\title{
LYRIC Indeterminate Response
}

National Cancer Institute

\section{Source}

National Cancer Institute. LYRIC Indeterminate Response. NCI Thesaurus. Code

C153156.

A lymphoma response that cannot be distinguished between flare/pseudo-progression and true progressive disease. 